\title{
Computation of Inertial Motion: Neural Strategies to Resolve Ambiguous Otolith Information
}

\author{
Dora E. Angelaki, ${ }^{1,2}$ M. Quinn McHenry, ${ }^{2}$ J. David Dickman, ${ }^{1,2}$ Shawn D. Newlands, ${ }^{1}$ and \\ Bernhard J. M. Hess ${ }^{3}$ \\ Departments of ${ }^{1}$ Surgery (Otolaryngology) and ${ }^{2}$ Anatomy, University of Mississippi Medical Center, Jackson Mississippi \\ 39216, and ${ }^{3}$ Department of Neurology, University Hospital, Zürich $\mathrm{CH}-8091$, Switzerland
}

According to Einstein's equivalence principle, inertial accelerations during translational motion are physically indistinguishable from gravitational accelerations experienced during tilting movements. Nevertheless, despite ambiguous sensory representation of motion in primary otolith afferents, primate oculomotor responses are appropriately compensatory for the correct translational component of the head movement. The neural computational strategies used by the brain to discriminate the two and to reliably detect translational motion were investigated in the primate vestibulo-ocular system. The experimental protocols consisted of either lateral translations, roll tilts, or combined translation-tilt paradigms. Results using both steady-state sinusoidal and transient motion profiles in darkness or near target viewing demonstrated that semicircular canal signals are necessary sensory cues for the discrimination between different sources of linear acceleration. When the semicircular canals were inactivated, horizontal eye movements (appropriate for translational motion) could no longer be correlated with head translation. Instead, translational eye movements totally reflected the erroneous primary otolith afferent signals and were correlated with the resultant acceleration, regardless of whether it resulted from translation or tilt. Therefore, at least for frequencies in which the vestibulo-ocular reflex is important for gaze stabilization $(>0.1 \mathrm{~Hz})$, the oculomotor system discriminates between head translation and tilt primarily by sensory integration mechanisms rather than frequency segregation of otolith afferent information. Nonlinear neural computational schemes are proposed in which not only linear acceleration information from the otolith receptors but also angular velocity signals from the semicircular canals are simultaneously used by the brain to correctly estimate the source of linear acceleration and to elicit appropriate oculomotor responses.

Key words: eye movements; vestibular; oculomotor; navigation; inertial; gravity; neural computation
In everyday life, one often experiences movements that involve both rotational and translational components. In addition, most naturally occurring rotational movements are accompanied by a concurrent change in head orientation relative to gravity. Running and locomoting, for example, have been shown to be characterized by high-frequency rotational and translational motion components (Grossman et al., 1988; Pozzo et al., 1990). Motion information is transduced by the vestibular system, which consists of separate receptors that respond to either angular (semicircular canals) or linear (otolith organs) accelerations. Because tilt and translation induce inertial accelerations of the otoconia that are physically equivalent (Einstein's equivalence principle; Einstein, 1908), primary otolith afferent neurons provide equivalent responses to both head tilts relative to gravity and to translational movements (Loe et al., 1973; Fernandez and Goldberg, 1976; Anderson et al., 1978; Dickman et al., 1991; Si et al., 1997). Thus, the otolith signals transmitted to the CNS merely encode the resultant linear acceleration $(\alpha)$, which is equal to the vectorial sum of the translational $(f)$ and gravitational $(g)$ components, i.e., $\alpha=f+g$.

Received July 2, 1998; revised Oct. 7, 1998; accepted Oct. 12, 1998.

This work was supported by National Institutes of Health Grant EY10851, Air Force Office of Scientific Research Grant F49620, and Swiss National Science Foundation Grant 31-47287.96.

Correspondence should be addressed to Dr. Dora Angelaki, Department of Surgery (Otolaryngology), University of Mississippi Medical Center, 2500 North State Street, Jackson, MS 39216-4505.

Copyright (C) 1998 Society for Neuroscience $\quad 0270-6474 / 98 / 190316-12 \$ 05.00 / 0$
Despite indiscriminate primary otolith afferent information, motor responses to head tilts and translations must be different. With respect to the oculomotor system, for example, a roll tilt of the head toward the shoulder should elicit ocular torsion, whereas a lateral head translation should generate horizontal eye movements (Bronstein and Gresty, 1988; Crawford and Vilis, 1991; Paige and Tomko, 1991a; Schwarz and Miles, 1991; Haslwanter et al., 1992; Tweed et al., 1994; Angelaki and Hess, 1996b; Telford et al., 1997; Angelaki, 1998). Because otolith afferent information does not discriminate between different sources of linear acceleration, it becomes the task of the CNS to correctly differentiate the acceleration source and to elicit functionally compensatory motor responses.

Two hypotheses have been proposed as to how the brain might solve the tilt-translation ambiguity of otolithic receptor information. According to the "multisensory integration" hypothesis, the brain must combine information from different sensors, such as the vestibular rotation sensors (i.e., the semicircular canals) and the otolith organs, to correctly differentiate between head translation and tilt (Guedry, 1974; Mayne, 1974; Young, 1974). Alternatively, the "frequency-segregation" hypothesis states that the frequency content of the otolith signals determines the source of acceleration. Accordingly, high-frequency accelerations are interpreted as translations, whereas low-frequency accelerations are interpreted as tilts (Paige and Tomko, 1991a; Telford et al., 1997).

In the present study, predictions derived from these hypotheses were tested. To directly investigate whether information from the 
semicircular canals is used by the CNS to correctly interpret linear accelerations, the horizontal eye movements elicited by linear acceleration before and after canal inactivation were taken as a measure of the capacity of the brain to encode head translation. The results refute frequency segregation as the primary computational scheme used to discern movement. Rather, functional semicircular canal signals are critical for an appropriate discrimination of the source of linear acceleration and the mode of head motion.

\section{MATERIALS AND METHODS}

\section{Animal preparation and eye movement recording}

Binocular three-dimensional (3-D) eye movements were recorded using the magnetic search coil technique. The driver coils, which generated horizontal and vertical magnetic fields (100 and $66 \mathrm{kHz}$, respectively) were mounted on a cubic fiberglass frame of 16-inch side length (CNC Engineering). Five rhesus monkeys (Macaca mulatta) were implanted with a head-restraining platform and dual search coils on each eye under gas anesthesia. Details for the surgical procedures have been described elsewhere (Angelaki, 1998). All surgeries were performed under sterile conditions in accordance with the Institutional Animal Care and Use Committee and National Institutes of Health guidelines. Anesthesia was initiated with an intramuscular injection of ketamine $(10 \mathrm{mg} / \mathrm{kg})$, followed by administration of an inhalative anesthesia that consisted of an $\mathrm{O}_{2}$-isoflurane mixture. Respiration, body temperature, and heart rate were continuously monitored. Animals were given antibiotics and analgesics after completion of each surgery. In addition, the lumens of all six semicircular canals were plugged in two of the animals, as described previously (Ewald, 1892; Angelaki et al., 1996). Canal-plugged animals showed no evidence of spontaneous nystagmus other than the normal downbeat nystagmus that is observed also in intact animals in darkness. After the surgery, animals were kept in complete darkness until the next morning when they were brought to the laboratory for vestibular testing ("acute" experimental protocol). After this acute vestibulo-ocular reflex (VOR) testing, animals were returned to the regular diurnal cycle.

The dual eye coil assembly that was implanted on each eye consisted of two serially interconnected miniature coils (Sokymat, Veveyse Switzerland) that were attached at diagonal points along the circumference of an $\sim 15 \mathrm{~mm}$ three-turn stainless steel coil (Cooner wire). The exact orientation of the two coils relative to each other and the orientation of the dual eye coil on the eye were precisely determined based on both preimplantation and daily calibration procedures (Hess 1990; Hess et al., 1992). Briefly, each dual eye coil was calibrated before surgical implantation with a 3-D calibration jig. Using rotations about all three axes, this calibration yielded the coil sensitivities, as well as the angle between the two coil sensitivity vectors. In each experimental session and protocol, pretrained animals performed a visual fixation task. The eye coil voltages measured during visual fixation, along with the precalibrated values for the sensitivity vector of the torsion coil and the angle between the two coils, were used to calculate the orientation of the dual coil on the eye, as well as offset voltages.

3-D eye positions were expressed as rotation vectors using straightahead gaze as the reference position. Angular eye velocity was computed from these rotation vectors (cf. Angelaki and Hess, 1996a,b,c). Both eye position and angular eye velocity vectors were expressed relative to a head-fixed right-handed coordinate system, with the head placed in an $18^{\circ}$ nose-down position (see below). Torsional, vertical, and horizontal eye position and velocity were defined as the components of the eye position and eye velocity vectors along the naso-occipital, interaural (IA), and vertical head axes, respectively. Positive directions were clockwise (as viewed from the animal, i.e., rotation of the upper pole of the eye toward the right ear), downward, and leftward for the torsional, vertical, and horizontal components, respectively.

\section{Experimental setup and protocols}

During experimental testing, the monkeys were seated in a primate chair, with their heads statically positioned such that the horizontal stereotaxic plane was tilted $18^{\circ}$ nose-down. This head position was used to place the lateral semicircular canals approximately parallel to the earth-horizontal plane, while at the same time keeping the vertical semicircular canals as vertically oriented as possible. The animal's body was secured with shoulder and lap belts, while the extremities were

\begin{tabular}{|c|c|c|c|c|}
\hline $\begin{array}{l}\text { Frequency } \\
(\mathrm{Hz})\end{array}$ & $\begin{array}{l}\text { Peak roll } \\
\text { amplitude }\end{array}$ & $\begin{array}{l}\text { Translation } \\
\text { amplitude }\end{array}$ & Phase $\left(^{\circ}\right)$ & IA Accel (G) \\
\hline $0.16 \mathrm{~Hz}$ & $5.7^{\circ}$ & $0.10 \mathrm{G}$ & $0^{\circ}, 180^{\circ}$ & $0.2, \quad 0.0$ \\
\hline $0.5 \mathrm{~Hz}$ & $21.8^{\circ}$ & $0.40 \mathrm{G}$ & $0^{\circ}, 180^{\circ}$ & $0.37,0.0$ \\
\hline $1.0 \mathrm{~Hz}$ & $5.0^{\circ}$ & $0.087 \mathrm{G}$ & $0^{\circ}, 180^{\circ}$ & $0.17,0.0$ \\
\hline
\end{tabular}

\section{Table 1B. Extended experimental protocols during combined} roll-translational stimuli

\begin{tabular}{llllll}
$\begin{array}{l}\text { Frequency } \\
(\mathrm{Hz})\end{array}$ & $\begin{array}{l}\text { Peak roll } \\
\text { amplitude }\end{array}$ & $\begin{array}{l}\text { Translation } \\
\text { amplitude }\end{array}$ & Phase $\left(^{\circ}\right)$ & $\begin{array}{l}\text { IA Accel } \\
(\mathrm{G})\end{array}$ & $\begin{array}{l}z \text { Accel } \\
(\mathrm{G})\end{array}$ \\
\hline $0.5 \mathrm{~Hz}$ & $21.8^{\circ}$ & $0.40 \mathrm{G}$ & $180^{\circ}$ & 0.00 & 1.08 \\
$0.5 \mathrm{~Hz}$ & $21.8^{\circ}$ & $0.30 \mathrm{G}$ & $180^{\circ}$ & 0.09 & 1.04 \\
$0.5 \mathrm{~Hz}$ & $21.8^{\circ}$ & $0.20 \mathrm{G}$ & $180^{\circ}$ & 0.19 & 1.00 \\
$0.5 \mathrm{~Hz}$ & $21.8^{\circ}$ & $0.10 \mathrm{G}$ & $180^{\circ}$ & 0.27 & 0.97 \\
$0.5 \mathrm{~Hz}$ & $21.8^{\circ}$ & $0.00 \mathrm{G}$ & $0^{\circ}$ & 0.37 & 0.93 \\
$0.5 \mathrm{~Hz}$ & $21.8^{\circ}$ & $0.10 \mathrm{G}$ & $0^{\circ}$ & 0.46 & 0.89 \\
$0.5 \mathrm{~Hz}$ & $21.8^{\circ}$ & $0.20 \mathrm{G}$ & $0^{\circ}$ & 0.56 & 0.85 \\
$0.5 \mathrm{~Hz}$ & $21.8^{\circ}$ & $0.30 \mathrm{G}$ & $0^{\circ}$ & 0.65 & 0.82 \\
$0.5 \mathrm{~Hz}$ & $21.8^{\circ}$ & $0.40 \mathrm{G}$ & $0^{\circ}$ & 0.74 & 0.77 \\
\hline $0.5 \mathrm{~Hz}$ & $16.9^{\circ}$ & $0.40 \mathrm{G}$ & $180^{\circ}$ & 0.09 & 1.04 \\
$0.5 \mathrm{~Hz}$ & $11.9^{\circ}$ & $0.40 \mathrm{G}$ & $180^{\circ}$ & 0.19 & 1.00 \\
$0.5 \mathrm{~Hz}$ & $6.8^{\circ}$ & $0.40 \mathrm{G}$ & $180^{\circ}$ & 0.27 & 0.97 \\
$0.5 \mathrm{~Hz}$ & $1.6^{\circ}$ & $0.40 \mathrm{G}$ & $180^{\circ}$ & 0.37 & 0.93 \\
$0.5 \mathrm{~Hz}$ & $3.7^{\circ}$ & $0.40 \mathrm{G}$ & $0^{\circ}$ & 0.46 & 0.89 \\
$0.5 \mathrm{~Hz}$ & $9.4^{\circ}$ & $0.40 \mathrm{G}$ & $0^{\circ}$ & 0.56 & 0.85 \\
$0.5 \mathrm{~Hz}$ & $15.3^{\circ}$ & $0.40 \mathrm{G}$ & $0^{\circ}$ & 0.65 & 0.82 \\
\hline $0.5 \mathrm{~Hz}$ & $21.8^{\circ}$ & $0.40 \mathrm{G}$ & variable & variable & variable
\end{tabular}

loosely tied to the chair. The primate chair was then secured inside the inner frame of a vestibular turntable, which consisted of a 3-D rotator on top of a linear sled powered by a servo-controlled linear motor $(2 \mathrm{~m}$ length; Acutronics Inc.). The two inner frames of the turntable were manufactured by nonmetallic composite materials to minimize interference with the magnetic fields. The 3-D turntable was used to deliver roll movements, whereas the linear sled was used to laterally translate the animals.

Experimental protocol 1. Steady-state sinusoidal oscillations at $0.16,0.5$, and $1 \mathrm{~Hz}$ were delivered in complete darkness (vergence angle of $\sim 1$ MA) (Angelaki, 1998). For each of these frequencies, four stimulus combinations of lateral translation and/or roll tilt were tested. The peak amplitudes during combined tilt-translational motion profiles are provided in Table $1 A$. As shown in Figure $1 A$ for $0.5 \mathrm{~Hz}$ stimuli, the Translation only stimulus consisted of translational displacements $( \pm 36.7$ $\mathrm{cm})$ along the animal's IA axis, with a peak IA shear acceleration of $0.37 \mathrm{G})$. In Figure $1 B$, the Roll tilt only stimulus consisted of earthhorizontal axis roll oscillations $\left( \pm 21.8^{\circ}\right)$, which generated the same IA shear acceleration of $0.37 \mathrm{G})$. In Figure $1 C$, the Roll tilt + Translation stimulus consisted of lateral linear translations (sled motion, $\pm 39.8 \mathrm{~cm}$ ) combined with simultaneous roll tilt oscillations $\left( \pm 21.8^{\circ}\right)$. The translational and tilt motions were produced in phase such that a total IA acceleration of $0.74 \mathrm{G}$ resulted. The Roll tilt - Translation stimulus in Figure $1 D$ consisted of the same combined translation and tilt components, except that the two motions were out of phase. This produced a total IA acceleration of $0.0 \mathrm{G}$.

Because the roll movements were nested inside the translational displacement, the translational acceleration along the IA axis differed slightly from that delivered by the linear sled. Specifically, if the roll oscillation amplitude is described by the equation $\theta(t)=\theta_{\mathrm{o}} \cdot \sin (\omega t)$, and the translational acceleration during linear motion is described by the equation $f_{\mathrm{tr}}=f_{\mathrm{o}} \cdot \sin (\omega t+\gamma)$, where $\gamma$ is the relative phase between the two stimuli, then during a combined motion profile, the translational 
(a)

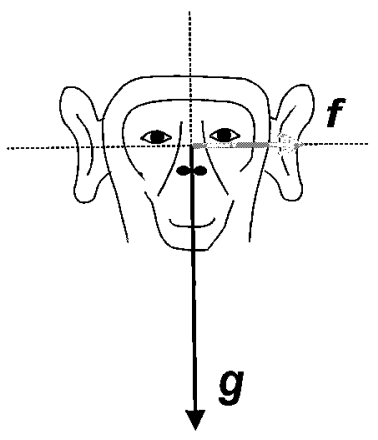

Translation only

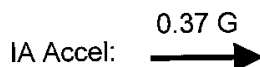

(c)

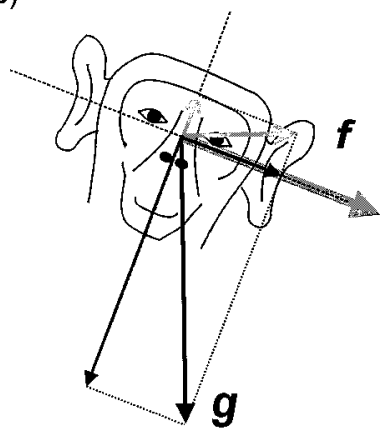

Roll tilt + Translation

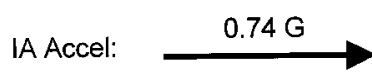

(b)

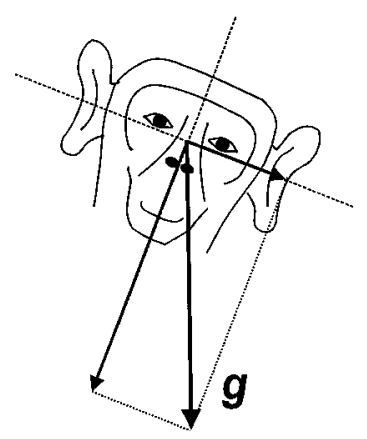

Roll tilt only

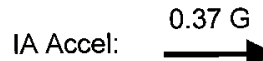

(d)

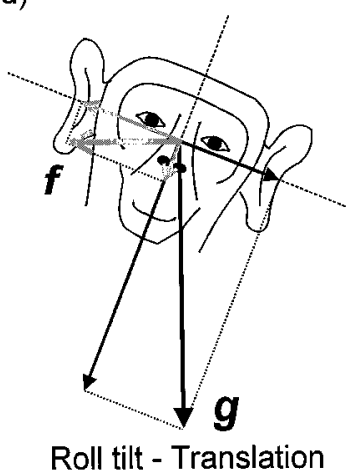

IA Accel: $\quad 0 G$

Figure 1. Schematic diagram outlining the main experimental protocol of lateral motion and/or roll tilt oscillations at $0.5 \mathrm{~Hz}$. $a$, Pure translation [black arrow, gravitational acceleration $(g)$; gray arrow, translational acceleration $(f)] . b$, Pure roll tilt. $c$, Combined roll tilt and translation with relative phases such that the translational component added to the gravity component along the IA axis, generating a resultant IA acceleration of 0.74G (thick arrow). $d$, Combined roll tilt and translation with relative phases such that the translational and gravitational components along the IA axis canceled each other (i.e., IA acceleration, 0G).

component of the acceleration along the IA axis $\left(f_{\mathrm{y}}\right)$ and the vertical head axis $\left(f_{\mathrm{z}}\right)$ would be:

$$
\begin{gathered}
f_{\mathrm{y}}=f_{\mathrm{o}} \cdot \cos (\theta(t)) \cdot \sin (\omega t+\gamma) \\
f_{\mathrm{z}}=-f_{\mathrm{o}} \cdot \sin (\theta(t)) \cdot \sin (\omega t+\gamma)
\end{gathered}
$$

Component $f_{\mathrm{y}}$ modulates sinusoidally at frequency $\omega$, whereas component $f_{\mathrm{z}}$ modulates at the second harmonic $(2 \omega)$. Vertical slow phase velocity modulation was indeed seen in the responses of intact animals (see Fig. 2). These vertical slow phase eye velocity components were partly attributable to a misalignment of the roll VOR and partly attributable to a second harmonic contribution because of the changing head orientation relative to the translational displacement (i.e., $f_{\mathrm{z}}$ in Eqs. $1 a, b)$. No such vertical eye movement components were elicited during combined tilt-translation in canal-plugged animals (see Fig. 5).

The total IA acceleration that results from simultaneous roll tilt and translation is given by the equation:

$$
\alpha_{\mathrm{y}}=f_{\mathrm{o}} \cdot \cos (\theta(t)) \cdot \sin (\omega t+\gamma) \pm \sin (\theta(t))
$$

Equations 1 and 2 have been used here to compute the translational and resultant acceleration components along the IA axis (see Fig. 9).

Experimental protocol 2. In addition to the four sinusoidal protocols

described above and illustrated in Figure 1, a more extensive tilttranslation combination battery of tests consisting of $0.5 \mathrm{~Hz}$ oscillations were also delivered (Table $1 B$ ). Specifically, (1) peak sled oscillation amplitude was kept constant $(0.40 \mathrm{G})$, whereas peak roll oscillation amplitude varied between $0^{\circ}$ and $\pm 21.8^{\circ} ;(2)$ peak sled oscillation amplitude was varied $(0-0.40 \mathrm{G})$, whereas peak roll oscillation amplitude was kept constant $\left(21.8^{\circ}\right)$; and finally, (3) the relative phase between the sled oscillations $(0.4 \mathrm{G})$ and roll oscillations $\left( \pm 21.8^{\circ}\right)$ was varied in $45^{\circ}$ steps. A phase of $0^{\circ}$ corresponded to the Roll tilt + Translation motion condition, whereas a phase of $180^{\circ}$ corresponded to the Roll tilt - Translation motion condition.

Experimental protocol 3. The same four main protocols (i.e., Translation only, Roll tilt only, Roll tilt + Translation motion, and Roll tilt Translation motion) were also tested with transient motion profiles. For these stimuli, the angular component of the stimulus consisted of a $15^{\circ}$ roll tilt (angular velocity, $60^{\circ} / \mathrm{sec}$; angular acceleration, $220^{\circ} / \mathrm{sec}^{2}$ ). The parameters of head translation were adjusted such that the inertial linear acceleration profile generated during translation closely matched that induced by the head tilt for the whole stimulus duration $(\sim 500 \mathrm{msec})$. As a result, the Roll tilt - Translation transients exhibited nearly zero IA acceleration.

Experimental protocol 4. For comparison, earth-horizontal and earthvertical axis roll oscillations were also delivered in a broader amplitude and frequency range. Specifically, the roll oscillation protocols in labyrinthine-intact animals included the following: $\pm 5^{\circ}(1 \mathrm{~Hz}) ; \pm 22^{\circ}$ $(0.5,0.2$, and $0.1 \mathrm{~Hz})$; and $\pm 90^{\circ}(0.2,0.1,0.05,0.02$, and $0.01 \mathrm{~Hz})$. Canal-plugged animals were only tested with the low-amplitude stimuli $\left(5^{\circ}\right.$ and $\left.22^{\circ}\right)$.

All steady-state sinusoidal recordings were made in complete darkness. Under these conditions, vergence averages were $\sim 1$ MA (Angelaki, 1998). Responses to transient stimuli were also recorded in complete darkness, but binocular fixation was controlled, and large vergence angles were obtained by initializing each trial only when the animal had satisfactorily fixated a target light located approximately in between the two eyes at a distance of $20 \mathrm{~cm}$. The target light was extinguished immediately before the onset of motion and remained off until the animal came to a complete stop. All animals participating in these experiments were pretrained using juice rewards to fixate targets paired with an auditory cue for variable time periods $(300-1000 \mathrm{msec})$ and then to maintain fixation after the target was turned off, for as long as the auditory tone was present (at least 1-2 sec). During all fixations, the room was illuminated (through small red lights) such that the animals could easily establish relative distance estimates of the targets. Adequate fixation was defined when both eyes were within behavioral windows (separate for each eye) of $\pm 1.0^{\circ}$. Animals were trained to perform these fixation tasks for at least 1 month before any experimental testing. As shown by the eye position traces of Figure 10, animals quickly learned to maintain vergence in complete darkness for a minimum of $500 \mathrm{msec}$, as long as the auditory tone remained on.

Responses were first obtained in animals with intact labyrinths. As stated above, acute responses were obtained during the first day after canal plugging (see Figs. 5, 10). Data were recorded up to 3 months after plugging. Because no changes were observed in the horizontal translational VOR properties after canal plugging (Angelaki, 1998, Fig. 7), data obtained at different times after plugging were included in the average (see Figs. 4, 6-9). The adequacy of canal plugging was physiologically verified by the lack of any response during earth-vertical axis yaw, pitch, and roll oscillations $(0.1-1 \mathrm{~Hz})$. These responses were systematically tested throughout the 3 month period of testing.

\section{Data analysis}

All data analyses were performed on personal computers off-line. First, calibrated 3-D eye positions were expressed as rotation vectors. The horizontal, vertical, and torsional components of the calibrated eye position vectors were then smoothened and differentiated with a Savitzky-Golay quadratic polynomial filter using a 15-point forward and backward window (Savitzky and Golay, 1964; Press et al., 1988). The angular eye velocity vector was computed from 3-D eye position and its derivative, as described previously (cf. Angelaki and Hess, 1996a,b,c). For transient motion profiles, only runs without fast phases for the first $500 \mathrm{msec}$ were further analyzed. For sinusoidal stimuli, fast eye movements were removed from the eye velocity profiles using a semiautomated procedure based on time and amplitude windows set for the second derivative of the magnitude of eye velocity. Subsequently, average response cycles were computed from steady-state response components 


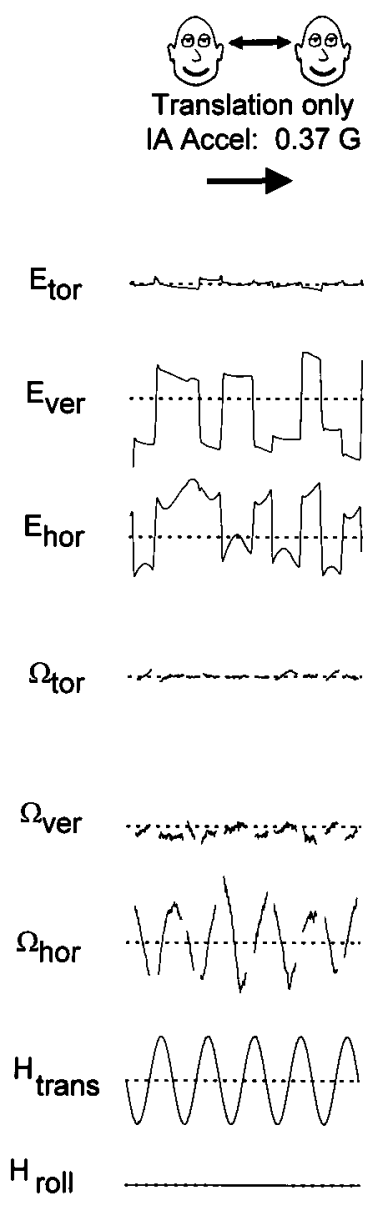

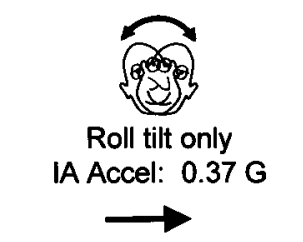

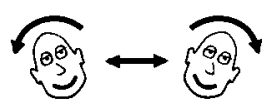
Roll tilt + Translation IA Accel: $0.74 \mathrm{G}$
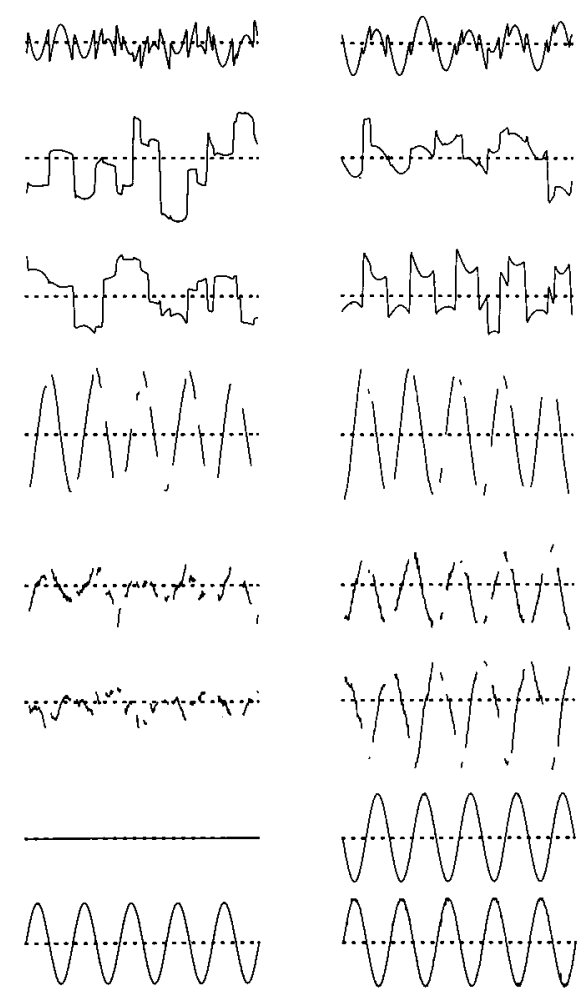

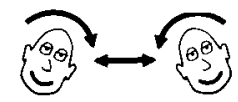

Roll tilt - Translation IA Accel: O G

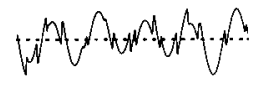

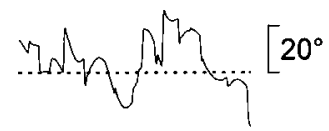
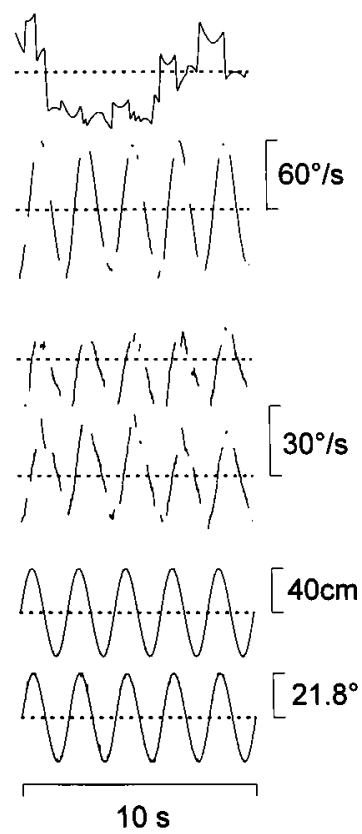

Figure 2. Tilt-translation discrimination in a labyrinthine-intact rhesus monkey. Torsional, vertical, and horizontal components of eye position $\left(E_{\text {tor }}\right.$, $E_{v e r}$, and $E_{h o r}$, respectively) and slow phase eye velocity $\left(\Omega_{t o r}, \Omega_{v e r}\right.$, and $\Omega_{h o r}$, respectively) of the right eye during lateral translation and/or roll tilt at 0.5 $\mathrm{Hz}$ in complete darkness. Left to Right, The stimuli consisted of Translation motion only, Roll tilt only, Roll tilt + Translation motion, and Roll tilt Translation motion. Dotted lines are zero position (straight-ahead gaze) and zero eye velocity. The stimulus traces (bottom) show sled position $\left(H_{\text {trans }}\right.$, positive direction to the left) and roll tilt position $\left(H_{\text {roll }}\right.$, positive tilt toward right ear-down). Positive eye movement directions are leftward, downward, and clockwise (upper pole of the eye toward the right ear).

(i.e., horizontal, vertical, and torsional) for each eye. For each of these average response cycles, peak response amplitude and phase were determined by fitting a sine function (including first and second harmonic, as well as a direct current offset) to both slow phase eye velocity and stimulus (angular or linear velocity) using a nonlinear least squares algorithm based on the Levenberg-Marquardt method. Phase was expressed as the difference (in degrees) between peak eye velocity and peak angular velocity (for roll tilt and tilt-translation combination profiles) or linear velocity (for pure translational motion).

The results presented here concentrate on horizontal eye movements, because they reflect the coding of head translation. In contrast, torsional eye movements were considered inappropriate in this regard, because they are primarily generated by semicircular canal activation, whereas static or otolith-induced eye torsion is known to have small gain in primates and humans (Diamond et al., 1979; Paige and Tomko, 1991a; Haslwanter et al., 1992; Angelaki, 1998).

Statistical comparisons on the data were based on ANOVA.

\section{RESULTS}

\section{Tilt-translation discrimination in labyrinthine-intact animals}

In all animals with intact labyrinths, the elicited eye movements were compensatory for all stimulus combinations. As shown in
Figure 2, for example, sinusoidal lateral translation (Translation only), but not roll tilt oscillations (Roll tilt only), elicited robust horizontal eye movements in complete darkness. Moreover, during the combined Roll tilt + Translation and Roll tilt - Translation motion profiles, the elicited horizontal eye movements were similar to those generated during Translation only motion. Thus, the horizontal eye movements generated in labyrinthine-intact animals totally ignored the linear accelerations caused by changes in head orientation relative to gravity during the roll movement. In fact, for all stimulus conditions (Translation only, Roll tilt only, Roll tilt + Translation, and Roll tilt - Translation motion protocols), the direction and magnitude of the horizontal response remained compensatory to the translational component of linear acceleration and was related neither to the acceleration produced by the roll tilt nor to the resultant IA acceleration. This was particularly striking for the Roll tilt - Translation protocols in which robust horizontal eye movements were generated, despite the fact that the resultant IA acceleration was zero.

As reported previously (Tweed et al., 1994; Angelaki and Hess, 

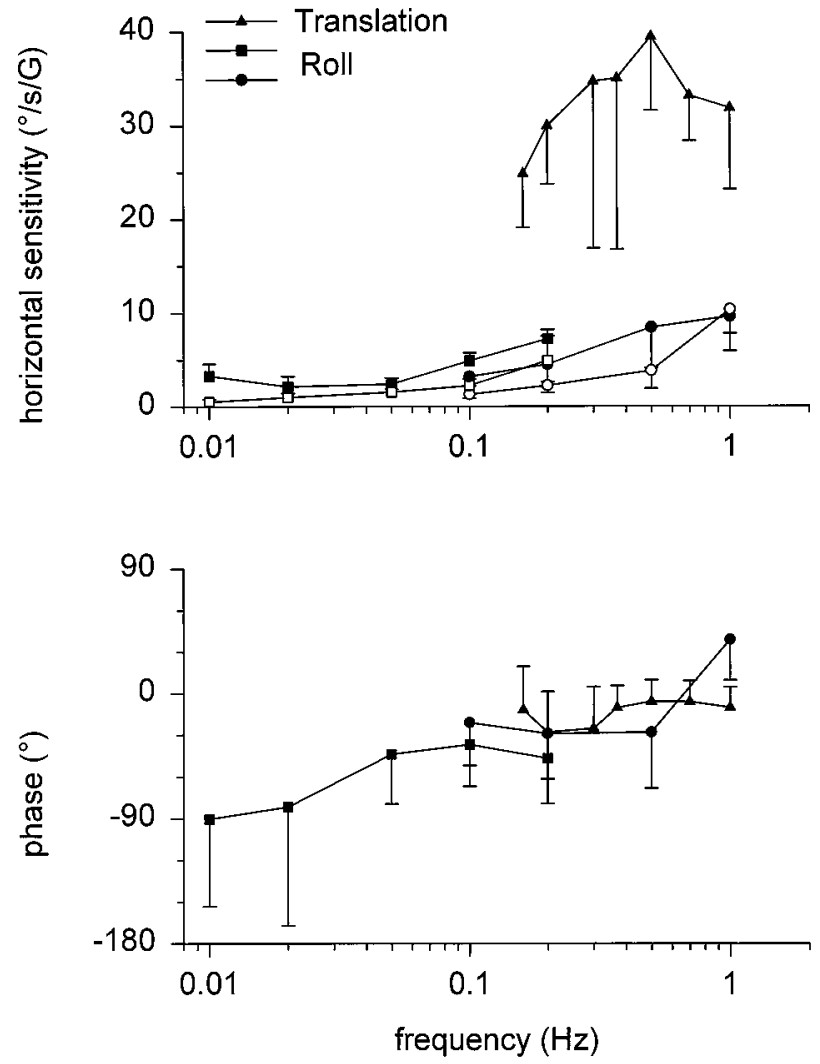

Figure 3. Comparison of horizontal eye velocity elicited during roll and translation. Mean \pm SD peak horizontal eye velocity sensitivity (expressed in degrees/second/gravity, where $G=9.81 \mathrm{~m} / \mathrm{sec}^{2}$ ) and phase have been plotted separately for earth-horizontal and earth-vertical roll oscillations (filled and open symbols, respectively). Roll oscillations: squares, $\pm 90^{\circ}(0.01-0.2 \mathrm{~Hz})$; circles, $\pm 22^{\circ}(0.1-0.5 \mathrm{~Hz})$ and $\pm 5^{\circ}(1 \mathrm{~Hz})$; lateral translations: triangles (Angelaki, 1998, Fig. 6, average data from five animals replotted).

1996b), a small horizontal eye velocity modulation was often present during roll head movements. This has been further quantified in Figure 3 in which sensitivity and phase of horizontal eye velocity during earth-horizontal and earth-vertical axis roll oscillations ( filled and open symbols, respectively) are compared with those generated during lateral translation (triangles) (Angelaki, 1998, Fig. 6). The horizontal eye movements elicited during both earth-horizontal and earth-vertical roll oscillations (i.e., rotations with the animals supine) were significantly smaller than those elicited during lateral head translation at the same frequency.

The observation that horizontal eye movements appropriately discriminated for tilt and translation is further illustrated in Figure 4 . In the frequency range tested, the results were independent of stimulus frequency $\left(0.16,0.5\right.$, and $1 \mathrm{~Hz} ; F_{(4,23)}=0.81 ; p>$ $0.05)$. Accordingly, robust horizontal eye movements were generated only in the presence of translational motion (Fig. 4, Tran only, Roll+Tran, Roll-Tran). In contrast, the horizontal eye movement components elicited during Roll only motion were significantly smaller in amplitude than those generated during Translation only motion $\left(F_{(1,15)}=68.0 ; p<0.05\right)$. During the combined Roll tilt + Translation and Roll tilt - Translation motion profiles, the horizontal eye movements were indistinguishable from those generated during Translation only motion $\left(F_{(2,23)}=\right.$ 2.1; $p>0.05)$. In fact, their amplitude and phase were always appropriate for gaze compensation during head translation.
Tilt/translation discrimination in labyrinthine-intact animals

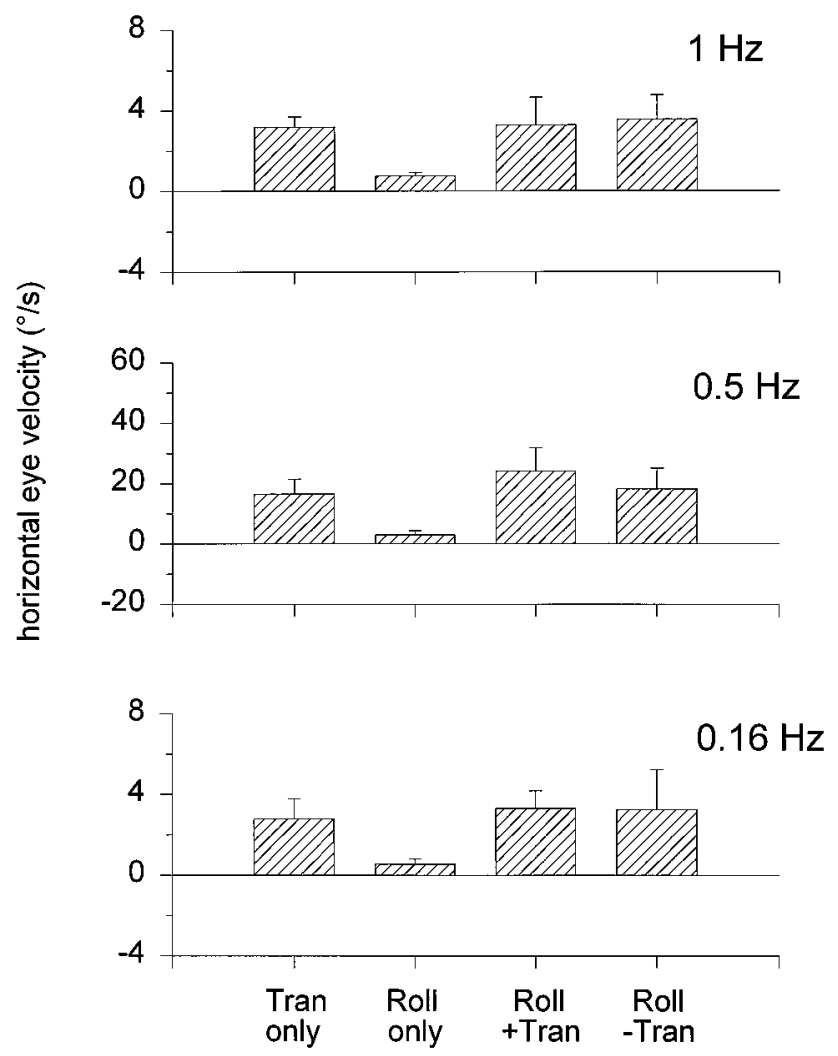

Figure 4. Translational (horizontal) VOR as a function of stimulus type in labyrinthine-intact animals. Mean \pm SD peak horizontal eye velocity from four monkeys with intact semicircular canals, tested at $1,0.5$, and $0.16 \mathrm{~Hz}$ (stimulus parameters are shown in Table $1 A$ ). Note that primate VOR correctly discriminates between tilt and translation at all tested frequencies.

\section{Tilt-translation discrimination in canal-plugged animals}

As shown in Figures 5 and 6, results were different in canalinactivated animals. Figure 5 illustrates responses from an animal acutely after canal plugging. Data from both canal-plugged animals have been summarized for all tested frequencies in Figure 6, $A$ and $B$, respectively. Responses to two stimuli were of particular interest. First, during Roll tilt only stimulation, a robust horizontal response component was observed in canal-plugged, but not labyrinthine-intact, animals. In fact, the horizontal responses in canal-plugged animals were indistinguishable during Translation only and Roll tilt only motions $\left(F_{(1,22)}=3.78 ; p>0.05\right)$. Second, horizontal responses differed for each of the Translation only, Roll tilt + Translation, and Roll tilt - Translation motion profiles $\left(F_{(2,32)}=115.5 ; \mathrm{p} \ll 0.05\right)$. Most noticeably, in the Roll tilt Translation stimulus condition, horizontal slow phase velocity was negligible, as was the resultant IA acceleration (Fig. 5).

\section{Different tilt-translation combinations}

To further corroborate these results, the tilt-translation combination protocols were expanded at $0.5 \mathrm{~Hz}$ in two different ways (Table 1B). First, peak roll oscillation amplitude was kept constant $\left(21.8^{\circ}\right.$, corresponding to a peak gravitational acceleration component of $0.37 \mathrm{G}$ along the IA axis), whereas peak sled oscillation amplitude varied. Second, peak sled oscillation amplitude was kept constant $(0.4 \mathrm{G})$, whereas peak roll oscillation amplitude 

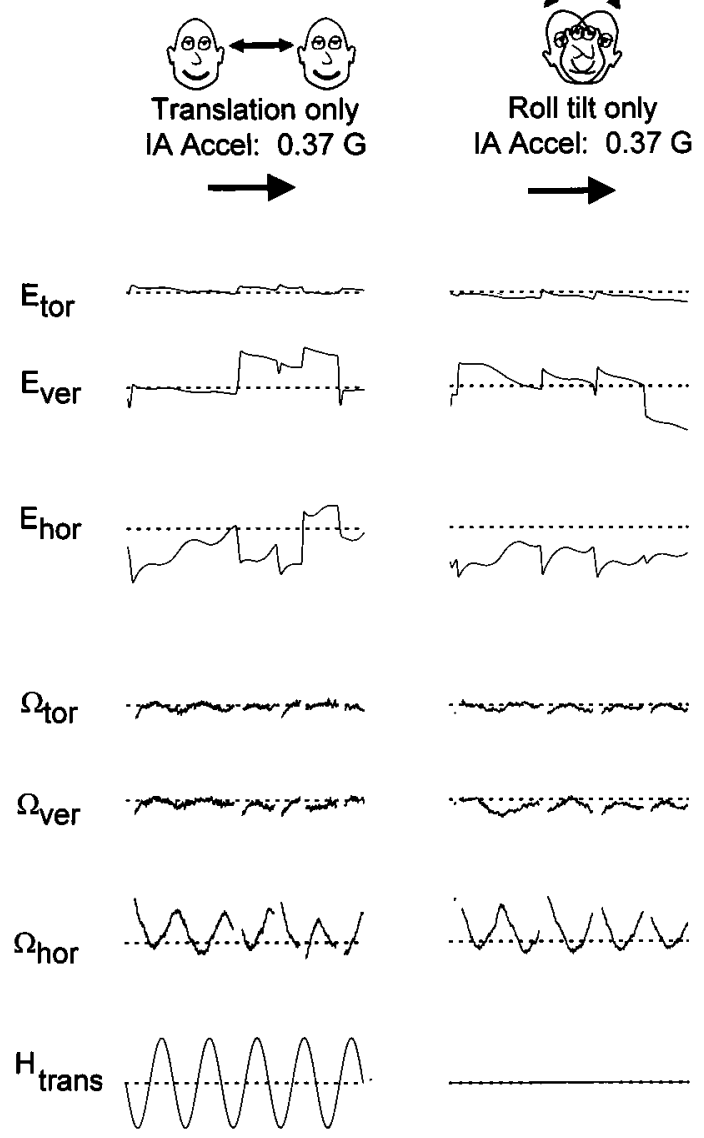

$\mathrm{H}_{\text {roll }}$

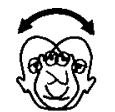

Roll tilt only

IA Accel: $0.37 \mathrm{G}$

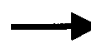

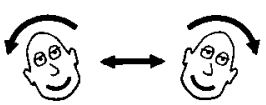

Roll tilt + Translation

IA Accel: $0.74 \mathrm{G}$

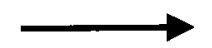

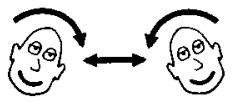

Roll tilt - Translation

IA Accel: O G
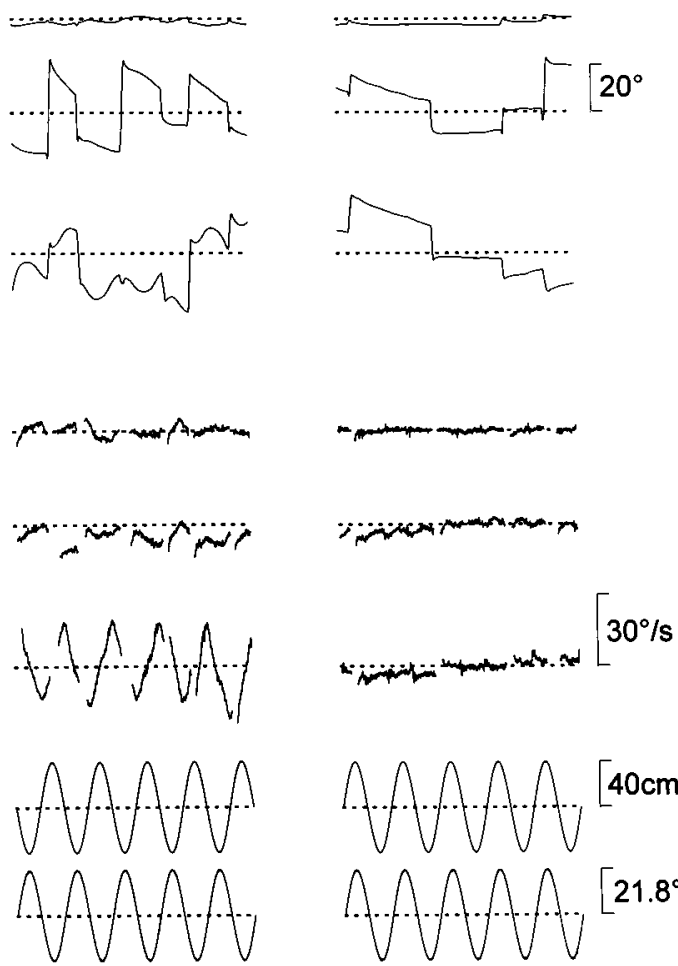

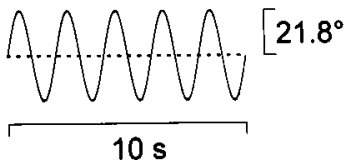

Figure 5. Tilt-translation discrimination after all semicircular canals were inactivated by plugging the canal lumen. Responses, stimuli, and figure organization as in Figure 2. Note that there is no horizontal response during Roll tilt - Translation motion (IA acceleration, 0G).
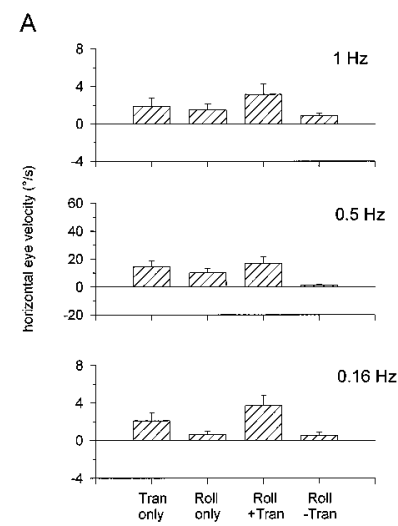

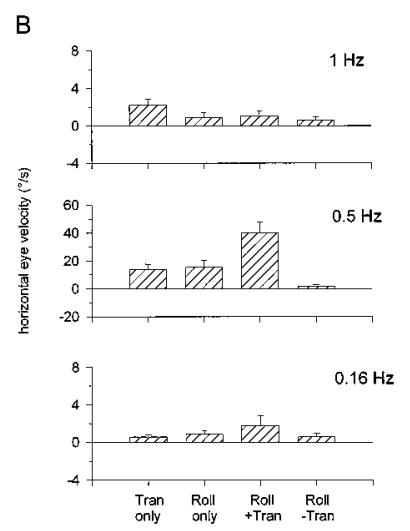

Figure 6. Translational (horizontal) VOR as a function of stimulus type in two canal-plugged animals. Mean \pm SD peak horizontal eye velocity elicited during lateral translation and/or roll tilt oscillations at $1,0.5$, and $0.16 \mathrm{~Hz}$. Notice the large differences when compared with labyrinthineintact animals (Fig. 4), particularly during Roll only and Roll tilt Translation stimuli. varied. In both sets of protocols, the resultant IA acceleration was identical, because it varied between 0 and $0.74 \mathrm{G}$ (in steps of 0.09G). Data from all three labyrinthine-intact and the two canalplugged animals tested have been summarized in Figures 7 and 8, $A$ and $B$ in both figures, respectively. As peak translational acceleration increased, peak horizontal eye velocity in labyrinthine-intact animals also increased in a linear manner, such that it was zero when $f_{\text {IA,peak }}$ was $0 \mathrm{G}$ (and IA acceleration was $0.37 \mathrm{G}$ ) and maximal (minimal) when $f_{\text {IA,peak }}$ was $\pm 0.37 \mathrm{G}$ (Fig. 7A). In contrast, as the peak gravitational acceleration component along the IA axis was changed and as long as translational acceleration remained the same, horizontal eye velocity in labyrinthine-intact animals remained constant and independent of peak roll oscillation amplitude (Fig. 8A). These data clearly demonstrate that the horizontal eye movements elicited during any combination of roll tilt and translation in labyrinthineintact primates precisely follows the translational rather than either the resultant or gravitational component of acceleration.

Very different observations were made in canal-plugged animals. As illustrated when Figures $7 B$ and $8 B$ are compared, horizontal eye velocity exhibited the same dependencies on linear acceleration, independently of the nature of the stimulus. In both combination protocols, horizontal eye velocity was zero only 
Combined roll/translational motion

(Roll: $21.8^{\circ}$; Translation: $0.0-0.37 \mathrm{G}$ )
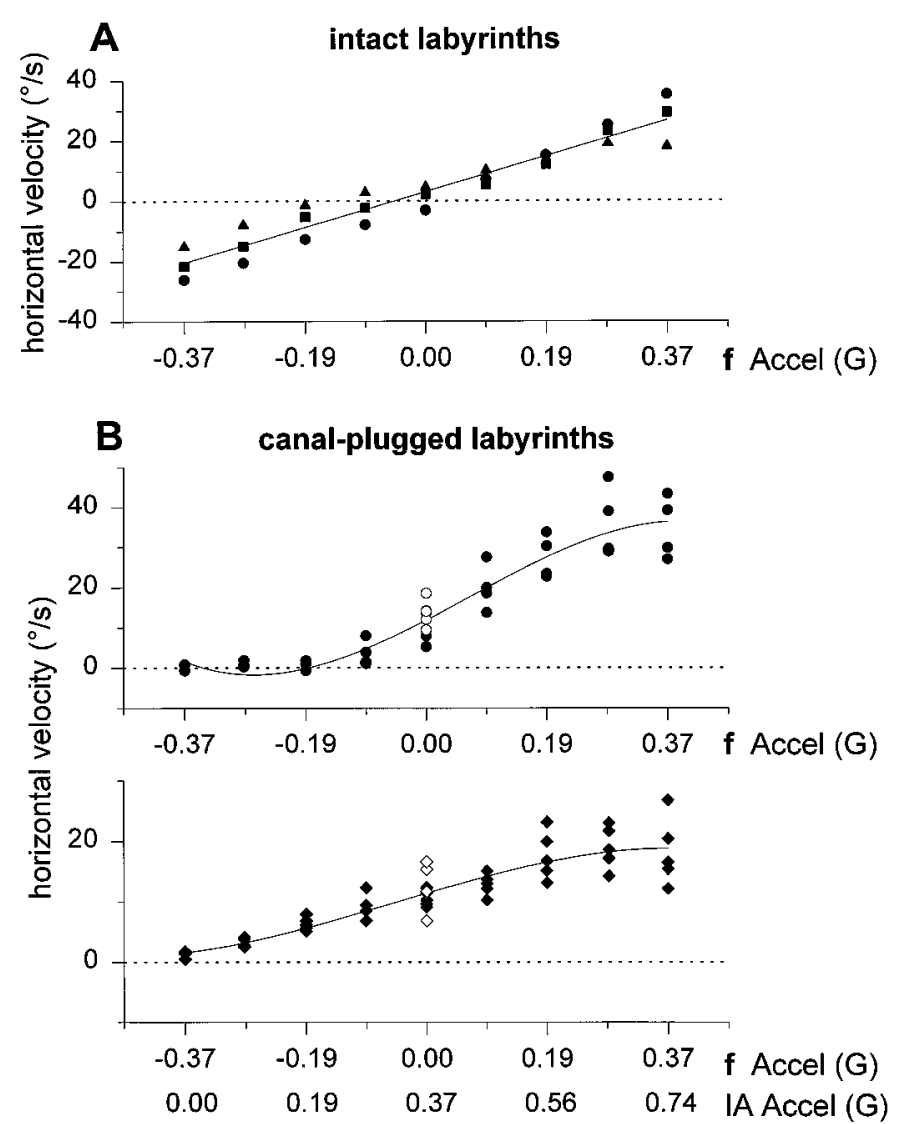

Figure 7. Combined roll-translational motion stimuli. Constant peak amplitude roll oscillations $\left(\theta_{\mathrm{o}}=21.8^{\circ}\right)$ were paired with varying peak amplitude and in phase or out of phase translational oscillations (Table $1 B)$. As peak translational acceleration amplitude $(f)$ was varied between 0 and $\pm 0.37 \mathrm{G}$, the resultant $\mathrm{IA}$ acceleration changed between 0 and $0.74 \mathrm{G}$. Horizontal slow phase velocity has been separately plotted for three intact $(A)$ and two canal-plugged $(B)$ animals (different symbols are used for different animals). Open symbols, Data obtained during pure translational motion $(0.37 \mathrm{G})$.

when the resultant IA acceleration was zero. Furthermore, the practically indistinguishable behavior of horizontal eye velocity in Figures $7 B$ and $8 B$ suggests that horizontal eye movements in canal-plugged animals are elicited in response to the resultant IA acceleration, with no functional distinction between tilt and translation. There was a nonlinear dependence of horizontal eye velocity on acceleration, exhibiting saturating characteristics at high IA acceleration levels. Horizontal eye velocity responses generated during pure translation (peak acceleration of $0.37 \mathrm{G}$ ) have been superimposed in Figure $7 B$ (open symbols). These data points fall approximately on the second order regression line drawn through the combination responses.

In a third protocol, the phase $(\gamma)$ of the translation relative to the roll tilt was varied. As shown in Figure $9 B$, amplitude and phase of horizontal eye velocity as a function of $\gamma$ was predicted to differ, depending on whether the relevant stimulus was translational acceleration $(f)$ or resultant IA acceleration (Eqs. 1, 2). Comparison of the observed peak horizontal eye velocity amplitude and phase as a function of the relative phase between tilt and translation strongly suggests different encoding of linear acceler-
Combined roll/translational motion

(Roll: $0^{\circ}-21.8^{\circ}$; Translation: $0.37 \mathrm{G}$ )
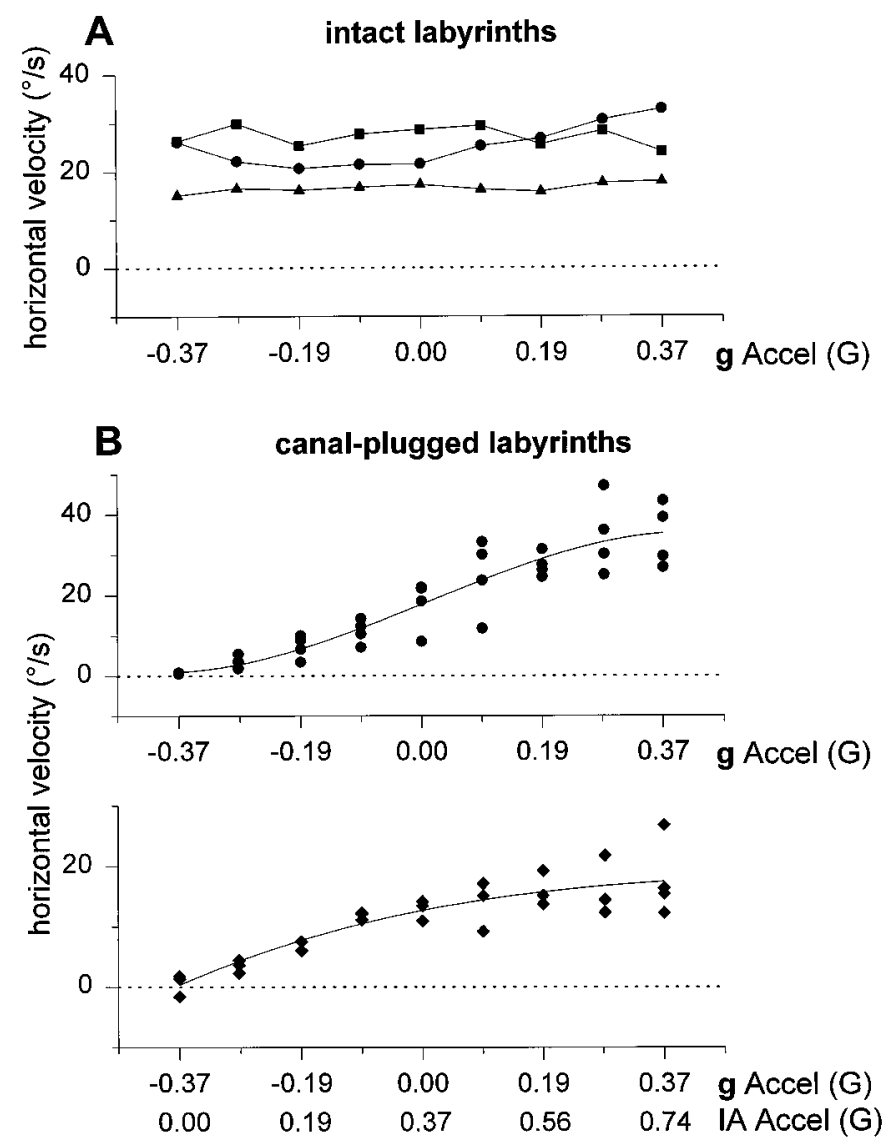

Figure 8. Combined roll-translational motion stimuli. Constant peak amplitude translational oscillations $\left(39.4 \mathrm{~cm}\right.$; i.e., $\left.f_{\mathrm{o}}=0.4 \mathrm{G}\right)$ were paired with varying peak amplitude, in phase or out of phase roll oscillations (Table $1 B$ ), such that the resultant IA acceleration varied between 0 and $0.74 \mathrm{G}$. Horizontal slow phase velocity has been separately plotted for three intact $(A)$ and two canal-plugged $(B)$ animals (different symbols are used for different animals).

ation in intact and canal-plugged animals. When semicircular canals were intact and functional, the peak horizontal eye velocity depended on relative phase according to a relationship that resembled the translational acceleration dependence. In contrast, horizontal eye velocity was clearly characterized by IA acceleration-dependent behavior when the semicircular canals were inactivated (Fig. 9A).

\section{Transient tilt-translation stimuli}

These observations regarding the role of semicircular canals in the correct discrimination of translational motion were independent of vergence angle. In fact, responses to transient head translation-roll tilt stimuli delivered as the animals maintained large vergence angles further corroborated these findings. Figure 10 plots binocular horizontal eye position and velocity recorded during transient motion profiles that were delivered immediately after fixation on a near, central visual target located $20 \mathrm{~cm}$ from the eyes (vergence of $\sim 10^{\circ}$ ). The stimulus consisted of a quick roll tilt toward the right ear combined with a transient lateral displacement to the right, which was associated with a nearly zero acceleration along the IA axis (Roll tilt - Translation condition) 


\section{Combined roll/translational motion}

A
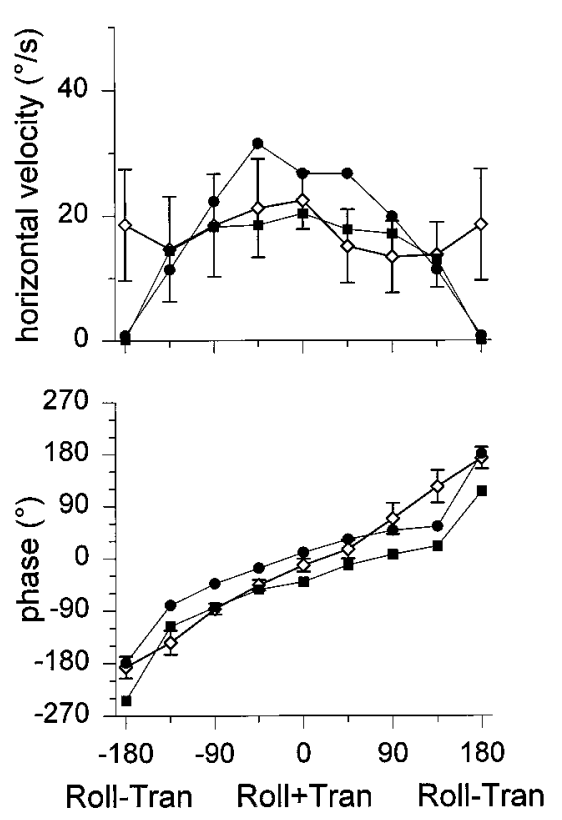

B
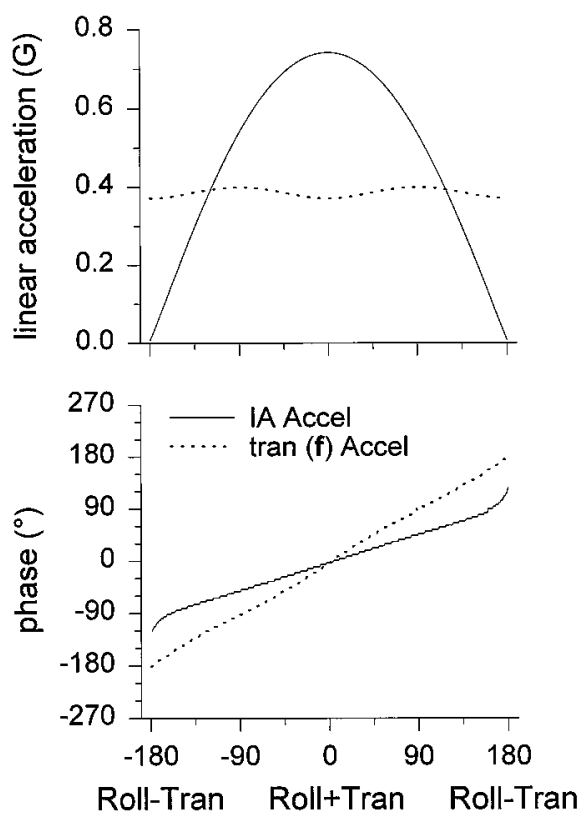

relative phase $\left({ }^{\circ}\right)$

Figure 9. Combined roll-translational motion stimuli. Constant peak amplitude roll oscillations $\left(\theta_{\mathrm{o}}=21.8^{\circ}\right)$ were paired with constant peak amplitude translational oscillations $\left(39.8 \mathrm{~cm}\right.$; i.e., $f_{\mathrm{o}}=$ $0.4 \mathrm{G})$ and variable phase. $A$, Mean \pm SD horizontal slow phase velocity amplitude and phase from intact animals (open circles) are compared with data from two canal-plugged animals (filled circles and squares). $B$, Theoretical predictions of the dependence of the resultant and the translational component of acceleration along the IA axis, according to Equations 1 and 2 (solid and dotted lines, respectively).
(Fig. 10). The elicited horizontal eye movements before and after semicircular canal inactivation clearly differed. Whereas robust leftward horizontal responses were elicited in the intact animal, negligible horizontal eye movements were observed in the same animal after all semicircular canals had been inactivated.

\section{DISCUSSION}

The problem of sensorimotor discrimination between inertial (translational) and gravitational components of linear acceleration has been a long-debated issue in motor control and inertial navigation. This study shows that not only otolith but also semicircular canal signals are necessary sensory cues for the appropriate discrimination between different sources of linear acceleration. Therefore, the oculomotor system discriminates between head translation and tilt primarily by sensory integration rather than frequency segregation of otolith afferent information.

\section{The central vestibular system correctly detects translational motion}

Because of the inherent ambiguity associated with afferent coding of linear acceleration, it has often been implied that the oculomotor system (and motor systems in general) is bound by similar limitations. Based on responses obtained during translational oscillations between 0.5 and $4 \mathrm{~Hz}$, for example, Paige and Tomko (1991a) and Telford et al. (1997) proposed that horizontal and torsional responses are always elicited simultaneously during both translation and tilt, as if responding to the resultant acceleration. Accordingly, otolith response ambiguity can only partially be resolved through central parsing of linear accelerations on the basis of frequency content. However, a direct test of this proposal was never performed.
Our results provide strong evidence in contrast to this interpretation, at least for the frequency range important for VOR compensation during movement (i.e., $>0.1 \mathrm{~Hz}$ ) (Angelaki, 1998). For all tilt-translation combination protocols, the direction and magnitude of the horizontal response of intact animals remained compensatory to the translational component of linear acceleration and was related neither to the acceleration produced by the roll tilt nor to the resultant IA acceleration. This was particularly striking for the Roll tilt - Translation protocols in which the resultant IA acceleration was zero, yet robust horizontal eye movements were generated.

\section{Inactivation of semicircular canals compromises accurate detection of translational motion}

The multisensory integration hypothesis predicts that both semicircular canal and otolith signals are needed for appropriate detection of the translational component of motion. In the absence of functional semicircular canal signals, correct motion discrimination would be compromised by interpreting the resultant IA acceleration as the translational stimulus. Conversely, if otolith signals were simply filtered, as the frequency-segregation hypothesis predicts, elimination of semicircular canal signals should have no effect on the ability of the vestibulo-ocular system to detect head translation.

The results from animals with inactivated semicircular canals clearly demonstrated that accurate discrimination between the translational and tilt components of motion was no longer possible. In fact, the elicited horizontal eye movements were always proportional to the resultant IA acceleration. The need for functional semicircular canal signals in the central processing of linear accelerations was further demonstrated by the experiments in which the relative phase of the two stimuli and either the trans- 

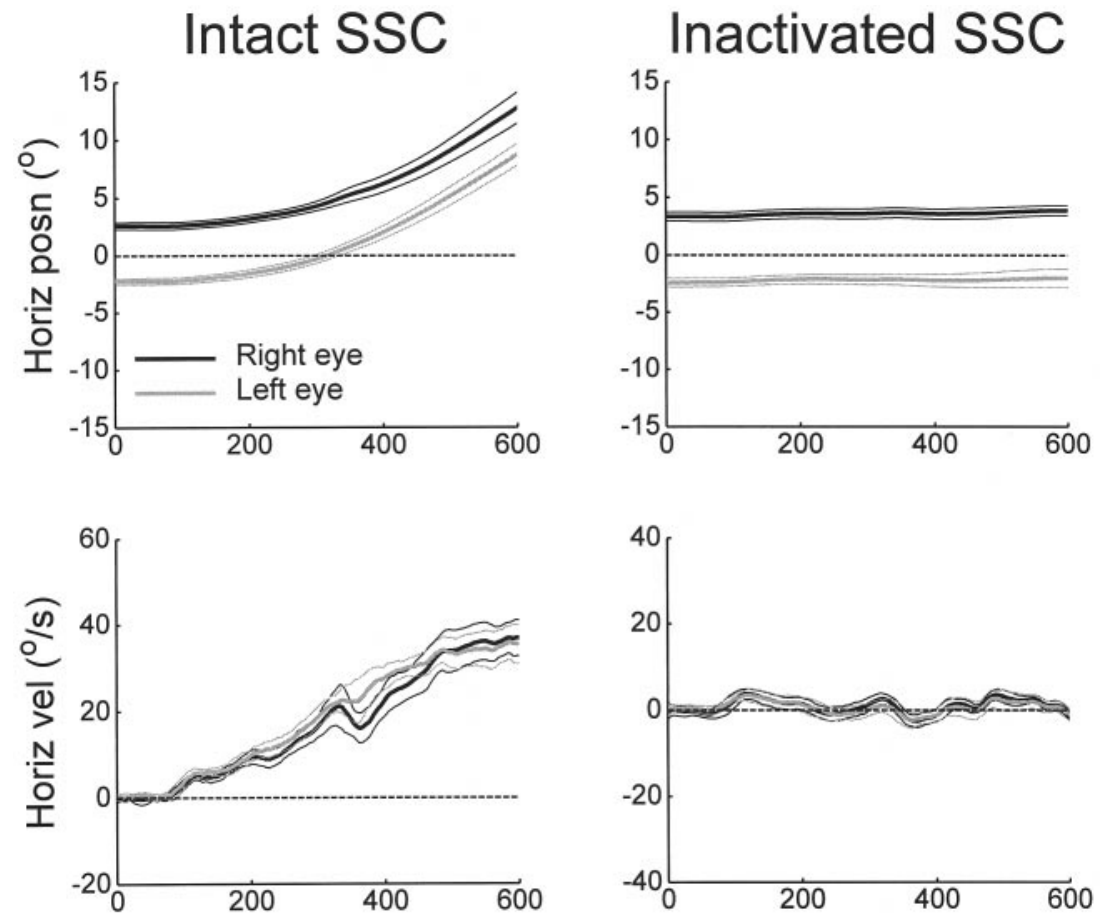

Figure 10. Transient Roll tilt - Translation motion profiles in an animal with intact and inactivated semicircular canals $(S C C)$. The stimulus consisted of a subtractive combination of a $15^{\circ}$ roll tilt toward the animal's right ear and linear translation to the right. Horizontal eye position (top) and eye velocity (middle) of both the right and left eyes (mean $\pm \mathrm{SD}$; left, nine trials; right, 26 trials). Dotted lines are zero eye position (straight-ahead gaze) and zero eye velocity. Bottom, Translational component and resultant IA acceleration measured as the outputs of two linear accelerometers mounted on the linear sled and on the animal's head, respectively. The IA acceleration trace was not measured for the Inactivated SCC plot and is therefore duplicated from the Intact SCC condition.

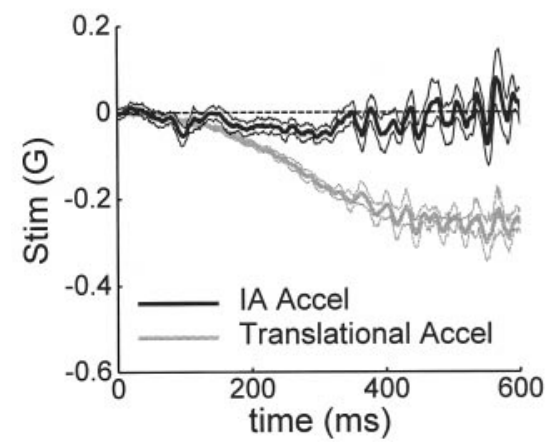

lational or the gravitational component of acceleration was varied separately (Figs. 7-9). Noticeably, a nonlinear dependence of horizontal eye velocity on IA acceleration amplitude was observed in the plugged animals (Figs. $7 B, 8 B$ ). A similar amplitude nonlinearity has been described in primary otolith afferents above 0.4-0.5G (Fernández and Goldberg, 1976). Interestingly, no such amplitude nonlinearity was seen in animals with intact labyrinths (Fig. 7A), although it is possible that primary afferents may have been driven into saturation.

Because translational VOR sensitivity strongly depends on viewing distance (Paige and Tomko, 1991b; Schwarz and Miles, 1991; Telford et al., 1997), the main stimulus combinations were also tested during short-lasting transient motion, immediately after satisfactory fixation on a near, centered target. The differences in the horizontal eye movements between labyrinthineintact and canal-plugged primates were even more provocative when viewing distance was controlled (Fig. 10). Despite robust horizontal eye movements during the Roll tilt - Translation motion profile during near target fixation in labyrinthine-intact animals, no horizontal translational response was generated in canal-inactivated animals when the resultant linear acceleration along the IA axis was zero. These results demonstrate that in the absence of functional signals from the semicircular canals and with all other sensory inputs essentially unchanged before and after canal inactivation, any linear acceleration component along the IA axis will be centrally interpreted as translational motion and generate a horizontal (translational) VOR.

\section{Nonlinear processing of vestibular afferent signals: neural computations to resolve tilt-translation ambiguity}

A fundamental question that arises from these results relates to the computational mechanisms used by the brain to discriminate the source of linear acceleration and to correctly detect head translation. Semicircular canal signals could contribute to the discrimination of head motion according to either one of the following principles. First, central integration of head angular velocity signals from the semicircular canals could be used to estimate the change in angular head orientation relative to gravity. For example, the inertial component that is caused by head translation (vector $f$ ) during a combined sinusoidal roll tilt plus translational motion could be extracted from the resultant otolith signal (vector $\alpha$ ) by estimating the roll motion via integration of 
the roll velocity signal [vector $\left.\omega=\left(\omega_{\mathrm{x}}, 0,0\right)\right]$, oriented along the head roll axis). Thus:

$$
f_{\mathrm{x}}=\alpha_{\mathrm{x}}=0, \quad f_{\mathrm{y}}=\alpha_{\mathrm{y}}-\sin (\theta(t)), \quad f_{\mathrm{z}}=\alpha_{\mathrm{z}}-\cos (\theta(t))
$$

with

$$
\theta(t)=\int_{0}^{\mathrm{t}} \omega_{\mathrm{x}}(\tau) d \tau
$$

describing roll tilt (re: gravity) as a function of time. In a more general case integration of 3-D angular velocity is more complex (e.g., Hess and Angelaki, 1997).

Second, a similar computational scheme utilizes angular velocity information from the semicircular canals (vector $\omega$ ), as well as static and dynamic linear acceleration information from otolith afferents (vectors $\alpha$ and $\dot{\alpha}=d \alpha / d t$ ). Based on the fact that the resultant linear acceleration is equal to the vectorial sum of translational and gravitational components, i.e., $\alpha=f+g$, and on the relationship $d g / d t=\dot{g}=\omega \times g$ (where $\times$ denotes the vector cross-product), which describes the rate of change of gravity, the translational component of acceleration $(f)$ can be easily computed by solving the following differential equation (Viéville and Faugeras, 1990; Hess and Angelaki, 1997):

$$
\dot{f}=\omega \times f+\dot{\alpha}-\omega \times \alpha
$$

Despite its apparent complexity, Equation 4 could be easily implemented in the CNS through signal convergence between angular velocity ( $\omega$, from semicircular canal afferents), as well as static and dynamic linear acceleration ( $\alpha$ and $\dot{\alpha}$, extracted from otolith afferents). The simplest neural network that could implement Equation 4 has been illustrated in Figure 11 and requires a minimum of four distinct "vector" neurons. The first two perform multiplicative interaction of their inputs, and the third is assumed to linearly summate its inputs. The fourth neuron functions as a neural integrator. Such computational steps have been often proposed to occur within single neurons or assemblies of neurons (Torre and Poggio, 1978; Koch et al., 1983; Shen, 1989). Furthermore, head angular velocity signals $(\omega)$, as well as both linear acceleration $(\alpha)$ and its time derivative $(\dot{\alpha})$ signals have been shown to coexist in single vestibular brainstem neurons (Angelaki et al., 1993).

These computational schemes and the conclusions reached based on the results of this study are only pertinent to frequencies

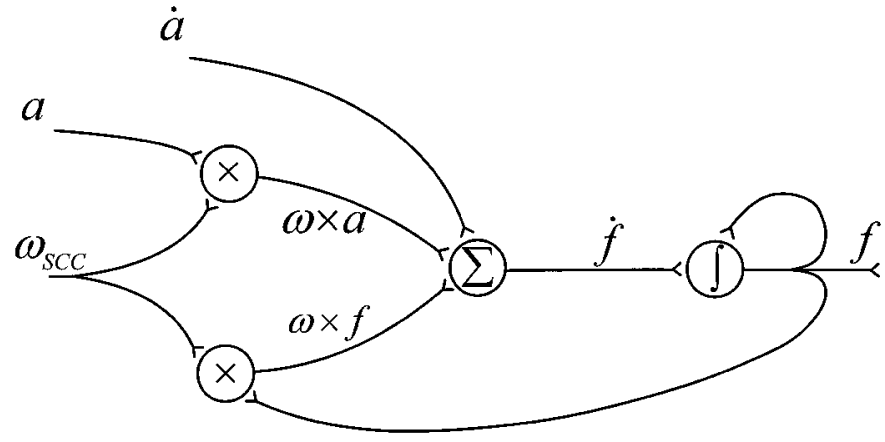

Figure 11. A four-neuron network, the simplest model that would implement the computations described by the differential Equation 4. $\omega_{S S C}$, Primary semicircular canal afferents; $\alpha, \dot{\alpha}$, static and dynamic otolith signals, respectively; $x, \Sigma, \int$, vector multiplication, summation, and signal integration, respectively.

higher than $0.1 \mathrm{~Hz}$ in which semicircular canal afferents can provide veridical information about head angular velocity. Below $\sim 0.05-0.1 \mathrm{~Hz}$, however, semicircular canal signals are no longer accurate detectors of head velocity. Several solutions and models have been proposed to account for low-frequency tilt-translational discrimination (e.g., Glasauer and Merfeld, 1997) and remain to be further tested experimentally.

\section{Detection of inertial motion: a fundamental sensorimotor task}

Living and moving in a gravitational field places particular computational demands on inertial motion estimation. Although the peripheral sensory transduction of motion is dictated and bound by the laws of physics, the brain can use multisensory information to interpret sensory afferent signals and compute inertial motion. Specifically, the issue of inertial navigation and movement control has been long recognized as a major computational task for both biological and man-made inertial guidance systems (Fernandez and Macomber, 1962; Barlow, 1964; Beritoff, 1965; Mayne, 1969a,b). The vestibular labyrinths, like inertial guidance systems, are equipped with a set of 3-D linear accelerometers (otolith organs) and a set of 3-D angular accelerometers with built-in integrators that accurately detect head angular velocity in a wide frequency range (semicircular canals). The challenging computational task arises when these two independent sets of signals get combined to compute motion in space. Accurate separation of the

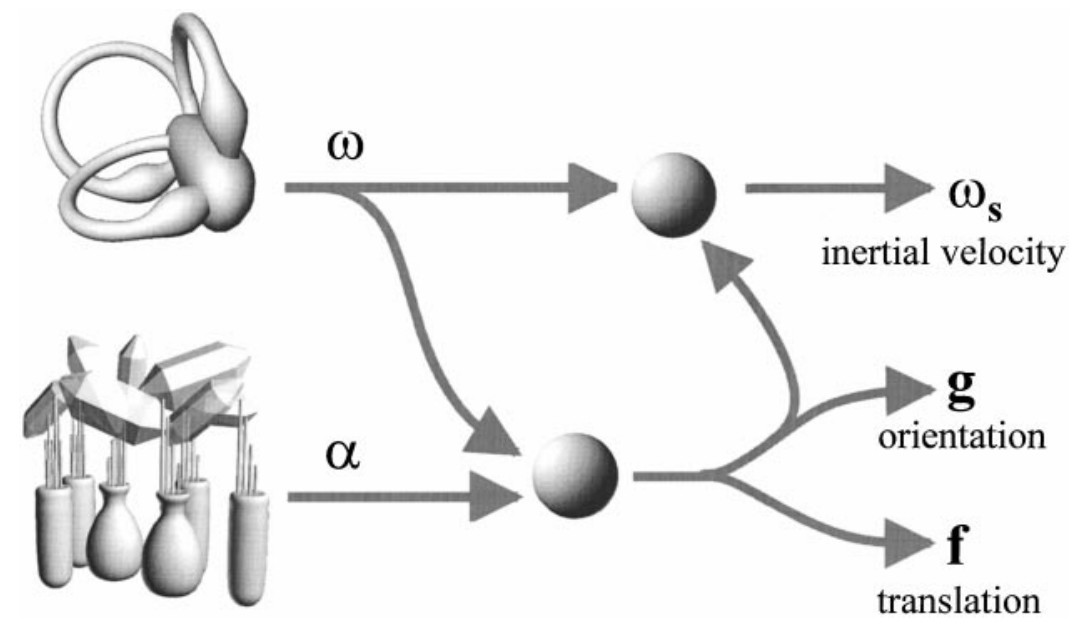

Figure 12. Schematic diagram summarizing the two main computations necessary to transform primary vestibular signals into inertial motion parameters. Angular velocity signals from the semicircular canals $(\omega)$ are used to segregate the resultant linear acceleration signals coded by primary otolith afferents $(\alpha)$ into gravitational ( $g$, orientation) and translational $(f)$ components. Gravitational estimates are also used to transform head-fixed angular velocity signals from the semicircular canals $(\omega)$ into inertial velocity, i.e., spacereferenced angular velocity $\left(\omega_{\mathrm{S}}\right)$ (Angelaki and Hess, 1994, 1995, 1996b). 
resultant acceleration $(\alpha)$ into the translational and gravitational components ( $f$ and $g$, respectively) comprises only one, but perhaps the most important, computational demand (Fig. 12). Inertial control of movement requires that, in addition, absolute angular motion in space be computed. Because primary semicircular canal afferents only code for a relative, head-fixed velocity vector $(\omega)$, estimation of inertial velocity $\left(\omega_{\mathrm{s}}\right)$ requires a second processing stage that uses the gravitational estimate $(g)$ to transform primary semicircular canal signals into space-referenced angular motion estimates (Fig. 12). Indeed, inertial velocity has been shown to be computed within the central vestibular system (Angelaki and Hess, 1994, 1995; Angelaki et al., 1995; Hess and Angelaki, 1997).

\section{APPENDIX}

Assuming a combined tilt-translation motion in which the roll oscillation amplitude is described as $\theta(t)=\theta_{\mathrm{o}} \cdot \sin (\omega t)$, and the translational acceleration during linear motion is described as $f_{\mathrm{tr}}$ $=f_{\mathrm{o}} \cdot \sin (\omega t+\gamma)$ where $\gamma$ is the relative phase between the two stimuli, the components of the gravitational and translation acceleration vectors in space- and head-fixed coordinates (Fig. 13) are as follows (in $\mathrm{G}$ units, $\mathrm{G}=9.81 \mathrm{~m} / \mathrm{sec}^{2}$ ):

Gravity vector $g$ :

in space-fixed coordinates:

$g_{\mathrm{x}}=0$

$g_{\mathrm{y}}=0$

$g_{\mathrm{z}}=-1$

in head-fixed coordinates (tilted by the angle $\theta$ relative to space):

$g_{\mathrm{x}}=0$

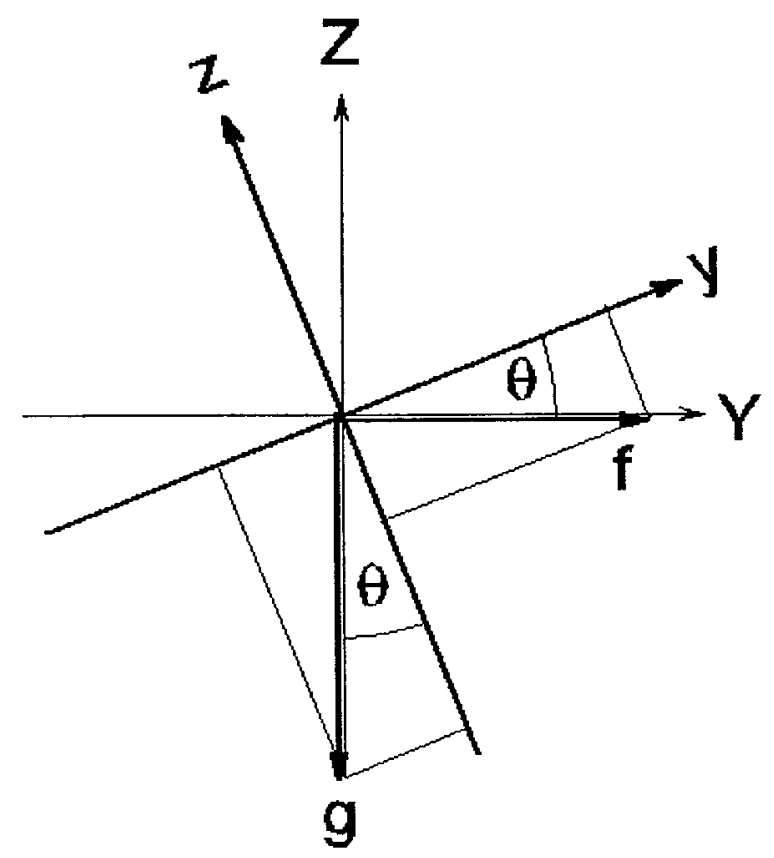

Figure 13. Tilt-translation paradigm. $x, y, z$, Head-fixed coordinates. $X$, $Y, Z$, Space-fixed coordinates. $\theta$, Roll tilt angle (about the $x$-axis).

$$
\begin{aligned}
& g_{\mathrm{y}}=-\sin (\theta) \\
& g_{\mathrm{z}}=-\cos (\theta)
\end{aligned}
$$

Translational acceleration $f$ : in space-fixed coordinates:

$f_{\mathrm{x}}=0$

$f_{\mathrm{y}}=f_{\mathrm{o}} \sin (\omega t+\gamma)$

$f_{\mathrm{z}}=0$

in head-fixed coordinates:

$f_{\mathrm{x}}=0$

$f_{\mathrm{y}}=f_{\mathrm{o}} \sin (\omega t+\gamma) \cos (\theta)$

$f_{\mathrm{z}}=-f_{\mathrm{o}} \sin (\omega t+\gamma) \sin (\theta)$

Acceleration $a$ (in head-fixed coordinates):

$a_{\mathrm{x}}=f_{\mathrm{x}}+g_{\mathrm{x}}=0$

$a_{\mathrm{y}}=f_{\mathrm{y}}+g_{\mathrm{y}}=f_{\mathrm{o}} \sin (\omega t+\gamma) \cos (\theta)-\sin (\theta)$ (resultant IA acceleration)

$a_{\mathrm{z}}=f_{\mathrm{z}}+g_{\mathrm{z}}=-f_{\mathrm{o}}(\omega t+\gamma) \sin (\theta)-\cos (\theta)$

Condition for cancellation of IA acceleration:

$a_{\mathrm{y}}=0=f_{\mathrm{o}} \sin (\omega t+\gamma) \cos (\theta)-\sin (\theta)$

Thus,

$\tan (\theta)=\tan \left(\theta_{\mathrm{o}} \sin (\omega t)\right)=f_{\mathrm{o}} \sin (\omega t+\gamma)$

approximation for small $\theta_{\mathrm{o}}: \tan \left(\theta_{\mathrm{o}}\right) \cong \theta_{\mathrm{o}} \cong \mathrm{f}_{\mathrm{o}}$

\section{REFERENCES}

Anderson JH, Blanks RHI, Precht W (1978) Response characteristics of semicircular canal and otolith systems in cat. I. Dynamic responses of primary vestibular fibers. Exp Brain Res 32:491-507.

Angelaki DE (1998) Three-dimensional organization of otolith-ocular reflexes in rhesus monkeys. III. Responses to translation. J Neurophysiol 80:680-695.

Angelaki DE, Hess BJM (1994) Inertial representation of angular motion in the vestibular system of rhesus monkeys. I. Vestibulo-ocular reflex. J Neurophysiol 71:1222-1249.

Angelaki DE, Hess BJM (1995) Inertial representation of angular motion in the vestibular system of rhesus monkeys. II. An otolithcontrolled transformation that depends on an intact cerebellar nodulus. J Neurophysiol 73:1729-1751.

Angelaki DE, Hess BJM (1996a) Three-dimensional organization of otolith-ocular reflexes in rhesus monkeys. I. Linear acceleration responses during off-vertical axis rotation. J Neurophysiol 75:2405-2424.

Angelaki DE, Hess BJM (1996b) Three-dimensional organization of 
otolith-ocular reflexes in rhesus monkeys. II. Inertial detection of angular velocity. J Neurophysiol 75:2425-2440.

Angelaki DE, Hess BJM (1996c) Adaptive modification of primate vestibulo-ocular reflex to altered peripheral vestibular inputs. II. Spatiotemporal properties of the adapted slow phase eye velocity. J Neurophysiol 76:2954-2971.

Angelaki DE, Bush GA, Perachio AA (1993) Two-dimensional coding of linear acceleration in vestibular nuclei neurons. $\mathrm{J}$ Neurosci 13:1403-1417.

Angelaki DE, Hess BJM, Suzuki J-I (1995) Differential processing of semicircular canal signals in the vestibulo-ocular reflex. J Neurosci 15:7201-7216.

Angelaki DE, Hess, BJM, Arai Y, Suzuki J-I (1996) Adaptation of primate vestibuloocular reflex to altered peripheral vestibular inputs. I. Frequency-specific recovery of horizontal VOR after inactivation of the lateral semicircular canals. J Neurophysiol 76:2941-2953.

Barlow JS (1964) Inertial navigation as a basis for animal navigation. J Theor Biol 6:76-117.

Beritoff JS (1965) Neural mechanisms of higher vertebrate behavior (Liberson WT, ed). Boston: Little, Brown.

Bronstein AM, Gresty MA (1988) Short latency compensatory eye movement responses to transient linear head acceleration: a specific function of the otolith-ocular reflex. Exp Brain Res 71:406-410.

Crawford JD, Vilis T (1991) Axes of eye rotation and Listing's law during rotations of the head. J Neurophysiol 65:407-423.

Diamond SG, Markham CH, Simpson NE, Curthoys IS (1979) Binocular counterrolling in humans during dynamic rotation. Acta Otolaryngol (Stockh) 87:490-498.

Dickman JD, Angelaki DE, Correia MJ (1991) Response properties of gerbil otolith afferents to small angle pitch and roll tilts. Brain Res 556:303-310.

Einstein A (1908) Über das Relativitätsprinzip und die aus demselben gezogenen Folgerungen. Jahrb Radioakt 4:411-462.

Ewald JR (1892) Physiologische Untersuchungen über das Endorgan des Nervus octavus. Wiesbaden, Germany: Bergmann.

Fernández C, Goldberg JM (1976) Physiology of peripheral neurons innervating otolith organs of the squirrel monkey. I. Response to static tilts and to long-duration centrifugal force. J Neurophysiol 39:970-984.

Fernandez M, Macomber GR (1962) Inertial guidance engineering. Englewood Cliffs, NJ: Prentice Hall.

Glasauer S, Merfeld DM (1997) Modelling three-dimensional responses during complex motion stimulation. In: Three-dimensional kinematics of eye, head and limb movements (Fetter M, Haslwanter T, Misslisch H, Tweed D, eds), pp 387-398. Amsterdam: Harwood Academic.

Grossman GE, Leigh RJ, Abel LA, Lanska DJ, Thurston SE (1988) Frequency and velocity of rotational head perturbations during locomotion. Exp Brain Res 70:470-476.

Guedry FE (1974) Psychophysics of vestibular sensation. In: Handbook of sensory physiology: the vestibular system, Pt 2, Psychophysics, applied aspects and general interpretations (Guedry Jr FE, ed). Berlin: Springer.

Haslwanter T, Straumann D, Hess BJM, Henn V (1992) Static roll and pitch in the monkey: shift and rotation of Listing's plane. Vision Res 32:1341-1348.

Hess BJ (1990) Dual search coil for measuring 3-dimensional eye movements in experimental animals. Vision Res 30:597-602.
Hess BJ, Angelaki DE (1997) Inertial vestibular coding of motion: concepts and evidence. Curr Opin Neurobiol 7:860-866.

Hess BJ, Van Opstal AJ, Straumann D, Hepp K (1992) Calibration of three-dimensional eye position using search coil signals in the rhesus monkey. Vision Res 32:1647-1654.

Koch C, Poggio T, Torre V (1983) Non-linear interactions in a dendritic tree: localization, timing and role in information processing. Proc Natl Acad Sci USA 80:2799-2802.

Loe PR, Tomko DL, Werner G (1973) The neural signal of angular head position in primary afferent vestibular nerve axons. J Physiol (Lond) 230:29-50.

Mayne R (1969a) The biological inertial system. Proc IEEE Resources Roundup 6:209-222.

Mayne R (1969b) The analogy of the vestibular organs to an inertial guidance system. In: Proceedings of the ninth international congress, Oto-Rhino-Laryngology, Mexico City, pp 407-415. Amsterdam: Excerpta Medica.

Mayne RA (1974) A systems concept of the vestibular organs. In: Handbook of sensory physiology: the vestibular system (Kornhuber HH, ed), pp 493-580. New York: Springer.

Paige GD, Tomko DL (1991a) Eye movement responses to linear head motion in the squirrel monkey. I. Basic characteristics. J Neurophysiol 65:1170-1182.

Paige GD, Tomko DL (1991b) Eye movement responses to linear head motion in the squirrel monkey. II. Visual-vestibular interactions and kinematic considerations. J Neurophysiol 65:1183-1196.

Pozzo T, Berthoz A, Lefort L (1990) Head stabilization during various locomotor tasks in humans. I. Normal subjects. Exp Brain Res 82:97-106.

Press WH, Teukolsky SA, Vettering WT, Flannery BP (1988) Numerical recipes in $\mathrm{C}$ : the art of scientific computing. New York: Cambridge UP.

Savitzky A, Golay MJE (1964) Smoothing and differentiation of data by simplified least squares procedures. Anal Chem 36:1627-1639.

Schwarz U, Miles FA (1991) Ocular responses to translation and their dependence on viewing distance. I. Motion of the observer. J Neurophysiol 66:851-864.

Shen L (1989) Neural integration by short term potentiation. Biol Cybern 61:319-325.

Si X, Angelaki DE, Dickman JD (1997) Response properties of pigeon otolith afferents to linear acceleration. Exp Brain Res 117:242-250.

Telford L, Seidman SH, Paige GD (1997) Dynamics of squirrel monkey linear vestibuloocular reflex and interactions with fixation distance. J Neurophysiol 78:1775-1790.

Torre V, Poggio T (1978) A synaptic mechanism possibly underlying direction selectivity to motion. Proc R Soc London Ser B 202:409-416.

Tweed D, Sievering D, Misslisch H, Fetter M, Zee DS, Koenig E (1994) Rotational kinematics of the human vestibuloocular reflex. I. Gain matrices. J Neurophysiol 72:2467-2479.

Viéville T, Faugeras OD (1990) Cooperation of the inertial and visual systems. In: Traditional and non-traditional robotic sensors (Henderson TC, ed). Berlin: Springer-Verlag.

Young LR (1974) Perceptions of the body in space: mechanisms. In: Handbook of physiology: The nervous system III, Chap 22, pp 1023 1066. 